\title{
Obtención de nuevos materiales híbridos orgánico-inorgánicos fotosensibles utilizables como láseres de colorante en estado sólido
}

\author{
O. GARCÍA 1 , A. COSTELA², I. GARCÍA-MORENO², C. GÓMEZ², R. SASTRE ${ }^{1}$ \\ 1 Instituto de Ciencia y Tecnología de Polímeros (C.S.I.C.), C/ Juan de la Cierva 3, 28006-Madrid. \\ 2 Instituto de Química-Física "Rocasolano", C/ Serrano 117-119, 28006-Madrid.
}

\begin{abstract}
La síntesis de nuevos materiales de carácter híbrido (orgánico-inorgánico) dopados con colorantes resultan altamente atractivos como medio activo en láseres de estado sólido ya que permiten combinar las excelentes propiedades térmicas que presentan los vidrios inorgánicos con las excelentes propiedades ópticas que presentan los materiales polímeros. Mediante un proceso simultaneo de policondensación sol-gel y de polimerización de la parte orgánica (monómero vinílico ó acrílico con grupos $\mathrm{OH}$ ), de forma que los grupos -OH del monómero orgánico participen también en el proceso de policondensación, hemos conseguido obtener monolitos válidos para su aplicación como láseres de colorante en estado sólido. Se ha llevado a cabo la síntesis y caracterización de nuevas matrices híbridas incorporando pirrometeno 567 como colorante láser (PM567); combinando polímeros de metacrilato de 2-hidroxietilo (HEMA) y copolímeros de HEMA y metacrilato de metilo (MMA) con diferentes proporciones en peso respecto al componente inorgánico (TEOS).

Los primeros resultados obtenidos con estos nuevos materiales demuestran que la presencia de pequeñas proporciones de TEOS en copolímeros de p(HEMA/MMA 1/1) aumentan la estabilidad térmica del colorante mejorando significativamente su acción láser. De hecho, algunos de los resultados alcanzados con estos nuevos materiales mejoran, significativamente, las fotoestabilidades alcanzadas para el PM567 incorporado en otras matrices inorgánicas e híbridas, así como en muchas de las matrices orgánicas seleccionadas como hospedadores para este colorante.
\end{abstract}

Palabras Clave: hibrido orgánico-inorgánico, polímero hibrido, láser de colorante en estado sólido, láser, pirrometeno 567.

\section{Synthesis of new photosensitive organic-inorganic hybrid materials used as solid state dye laser}

The synthesis of new materials of hybrid character (organic-inorganic) doped with dyes results highly attractive like active medium in solid state laser in which the excellent thermal properties of the inorganic glasses with the excellent optic properties of the polymeric materials could be combined. Through a simultaneous synthesis process of sol-gel policondensation of the inorganic TEOS during the free radical polymerization of the organic part (vinylic or acrylic monomers with $\mathrm{OH}$ groups), since the presence of this -OH side groups in the organic monomer can participate in the policondensation process of silanols, hybrids monoliths were able to be obtained as solid-state dye laser matrices. The synthesis and characterization of new hybrid matrices were carried out incorporating pirromethene 567 (PM567) into polymers of 2-hydroxyethyl methacrylate (HEMA) and copolymers of HEMA and methyl methacrylate (MMA) with different weight proportions of the inorganic component, tetraethoxysilane (TEOS). The first results obtained with this new materials are indicave that the addition of low proportions of TEOS into the polymer P(HEMA/MMA 1/1) increases its thermal stability reducing the thermal degradation of the dye and, consequently, improving significantly its laser action. In fact, some of the results achieved with these new materials represent the best photostability reached to date for PM567 incorporated into inorganic and hybrid materials and, taking into account the experimental pumping conditions, improve or at least, compare well, with the lasing properties of the dye embedded in other host polymeric matrices.

Key words: organic-inorganic hybrid, hybrid polymer, solid-state dye laser, laser, pyrromethene 567.

\section{1.- INTRODUCCIÓN}

En los últimos años se ha venido prestando un gran esfuerzo investigador dirigido al desarrollo de nuevos materiales fotosensibles con propiedades ópticas y optoelectrónicas debido, principalmente, a su enorme trascendencia técnico-económica. Existen, dentro de este campo, toda una serie de nuevas aplicaciones industriales basadas en polímeros fotosensibles, algunas de ellas presentes en nuestra vida cotidiana (1-6).

Asimismo, el estudio de nanomateriales orgánico-inorgánicos empieza a ser un campo de investigación en expansión. Estos nuevos materiales, considerados como materiales avanzados, prometen nuevas aplicaciones en distintos campos (7-9), potenciadas por sus propiedades físicas, particularmente ópticas, debido a la reducción en el tamaño de los dominios que los componen, que puede variar desde la escala del nanómetro (nanocomposites) hasta el subnanómetro (composites moleculares); de forma que, mediante la incorporación de fragmentos orgánicos entre, por ejemplo, óxidos inorgánicos, es posible diseñar nuevos materiales híbridos con propiedades que pueden diferir de aquellas que poseen sus componentes individualmente.

Hoy en día existen procedimientos que permiten que dos componentes inherentemente incompatibles (polímeros orgánicos y óxidos inorgánicos), se hagan compatibles por pre-mezcla de ambos a escala molecular antes de su conversión en un nuevo material $(10,11)$. La viabilidad de estos procedimientos se debe, en parte, al hecho de que los átomos de silicio formen enlaces estables, tanto con átomos de oxígeno como con átomos de carbono. Esto permite el diseño y la síntesis de materiales híbridos avanzados con propiedades físico-químicas muy específicas $(12,13)$. Las propiedades físicas de estos nanocomposites están normalmente potenciadas por la reducción del tamaño de sus dominios, de forma que cuanto más se reduce el tamaño de los mismos más se incrementa el área de superficie interfacial. En los composites 
moleculares no existe una separación de fases entre la fase orgánica e inorgánica; así, ciertos cristales híbridos conteniendo funcionalidades orgánicas pueden exhibir buena homogeneidad y alta transparencia. Este concepto es importante para la fabricación de materiales ópticos basados en materiales híbridos.

Los materiales de gel de sílice presentan, en principio, mejores propiedades térmicas que los polímeros, y la incorporación de colorantes a matrices inorgánicas mediante las técnicas sol-gel ha permitido la obtención de materiales fotoestables y con aceptable eficiencia láser $(14,15)$. No obstante, recientemente se han obtenido nuevos polímeros modificados con umbrales de daño respecto a la radiación láser tan altos como los de los materiales inorgánicos, y con estabilidades comparables o superiores a las de los geles de sílice bajo las mismas condiciones experimentales (16-18). Los polímeros presentan, además, otras ventajas importantes sobre los geles de sílice, tales como una mayor capacidad para disolver los colorantes orgánicos, una homogeneidad óptica superior (19) (lo que es extremadamente importante para el desarrollo de osciladores láser de pequeña anchura de línea) y una adaptabilidad a técnicas de fabricación económicamente más favorables (lo que facilitaría la miniaturización y el diseño de sistemas ópticos integrados). No menos importante es la relativa facilidad con la que se puede modificar la estructura y la composición química de los polímeros, lo que permite introducir cambios controlados para optimizar sus propiedades, con vistas a su utilización en una aplicación concreta (20).

Teniendo en cuenta todas estas consideraciones, nos planteamos la posibilidad de sintetizar nuevos materiales de carácter híbrido (orgánico-inorgánico) que nos permitieran combinar las excelentes propiedades térmicas que presentan los vidrios inorgánicos con las excelentes propiedades y ventajas que presentan los materiales polímeros. Así, se ha llevado a cabo la síntesis simultanea de nuevas matrices híbridas de metacrilato de metilo (MMA) y metacrilato de 2-hidroxietilo (HEMA) como componente orgánico, con diferentes proporciones en peso de tetraetoxisilano (TEOS) como componente inorgánico, a las que se les incorporó el pirrometeno 567 (PM567), como colorante, debido a la excelente eficiencia láser que presenta este colorante tanto en líquido como en sólido $(16,21,22)$ y la buena estabilidad que presenta en materiales polímeros $(22,23)$.

\section{2.- PARTE EXPERIMENTAL}

\section{1.- Materiales.}

Pirrometeno 567 (Excitón, grado láser), tetraetoxisilano (TEOS) (ABCR, pureza de $99 \%$ ) y ácido clorhídrico $(35 \%)(\mathrm{HCl})$ de Panreac, fueron empleados según se recibieron.

Metacrilato de metilo (MMA) y metacrilato de 2-hidroxietilo (HEMA), ambos de Aldrich-99\% pureza, fueron destilados y secados sobre tamiz $4 \AA ̊$ antes de su utilización. El iniciador: $\alpha, \alpha^{\prime}$-azo-bis-isobutironitrilo (AIBN) de Aldrich se recristalizó en etanol antes de su uso.

\section{2.-Síntesis de los monolitos híbridos.}

Se han sintetizado distintos monolitos híbridos (orgánico-inorgánicos) mediante el proceso de preparación sol-gel. Para ello se llevó a cabo la polimerización simultánea de la parte orgánica (monómero metacrílico con grupos $\mathrm{OH}$ ) y de la parte inorgánica (TEOS), de forma que los grupos -OH del monómero orgánico participen también en el proceso de policondensación (24).

La polimerización sol-gel del alcóxido metálico (TEOS) se ha catalizado añadiendo, bajo agitación constante, a la mezcla de polimerización agua $\left(\mathrm{H}_{2} \mathrm{O}\right)$ y ácido clorhídrico $(\mathrm{HCl})$, manteniendo en todos los casos las siguientes proporciones molares: [HCl] / [TEOS] $=1,8510^{-2}$ y $\left[\mathrm{H}_{2} \mathrm{O}\right] /[\mathrm{TEOS}]=2$. El contenido total del precursor ha sido ajustado en función de las proporciones con mayor contenido en silicio que permitían la síntesis de monolitos híbridos en perfectas condiciones, es decir, sin fragmentarse y manteniendo sus propiedades ópticas (transparencia, color...); en nuestro caso este contenido ha variado desde $1 \%$ p hasta un máximo del $30 \%$ p de TEOS.

Por otro lado, la polimerización de la parte orgánica del híbrido, en todos los casos, se ha llevado a cabo por polimerización radicálica en bloque empleando un iniciador térmico $\alpha, \alpha^{\prime}$-azo-bis-isobutironitrilo (AIBN), en la concentración adecuada $(0,5 \% \mathrm{p})$ respecto a la cantidad total de monómero presente en la mezcla de polimerización. Dicho iniciador fue seleccionado ya que los radicales primarios iniciadores, generados quedan como grupos finales en la cadena del polímero, son transparentes a las longitudes de onda de excitación y emisión empleadas durante el proceso de generación de luz láser. Es en esta fase orgánica donde se incorporó el colorante en proporción adecuada $(1,5 \mathrm{mM})$. Como es bien conocido, los colorantes derivados de estructuras pirrometénicas pierden con facilidad su actividad láser en condiciones de medio ácido, de forma que tras una media hora de agitación, a la mezcla de monómero-iniciador se le añaden pequeñas cantidades de piridina hasta conseguir que la mezcla disminuya su acidez hasta alcanzar $\mathrm{pH}>5$. Posteriormente, la mezcla conteniendo monómero-iniciador-colorante en disolución verdadera se añade, gota a gota y bajo agitación, a la disolución ya hidrolizada de TEOS, manteniéndose, de nuevo, la agitación vigorosa de la mezcla final al menos otros 30-60 minutos.

Por último, la mezcla híbrida resultante es vertida en moldes cilíndricos de polipropileno ( $14 \mathrm{~mm}$ de diámetro), con el fin de obtener una configuración geométrica próxima a la que se desea que tenga el material híbrido como medio activo del láser de colorante. Los moldes se introducen en un baño termostatizado a 30-40C o bien en una estufa a $45 \mathrm{C}$ durante 1-2 meses. El programa de temperaturas se ha adecuado a cada caso particular en función del grado de polimerización alcanzado, pero en todos ellos la temperatura se va aumentando lentamente, desde la temperatura inicial seleccionada hasta alcanzar los $80 \mathrm{C}$, para descender luego progresivamente hasta temperatura ambiente.

En todos los casos, el volumen final de disolución preparada para la polimerización se ha ajustado a $6 \mathrm{~mL}$, ya que durante el proceso de polimerización y secado se produce una significativa contracción de volumen en los monolitos obtenidos, en algunos casos superior al $40 \%$.

Siguiendo este procedimiento experimental se ha llevado a cabo la síntesis de diferentes matrices híbridas en forma de monolitos sólidos de $3-4 \mathrm{~cm}$ de altura, ópticamente transparentes y con buenas propiedades mecánicas.

\section{3.- Metodología}

Tras ser desmoldada una muestra, se obtiene en forma aproximadamente cilíndrica. Sin embargo, tanto en la superficie como en el núcleo del material, en algunos casos, pueden existir defectos como burbujas, rugosidad superficial, etc. y sobre todo, en el caso de estos materiales híbridos, fracturas. Todos estos defectos son originados por la contracción que sufre el material durante el proceso de polimerización y su posterior enfriamiento, por lo que en cualquier caso, se hace necesario un proceso de mecanizado y pulido de la muestra, previamente a su utilización.

La muestra se mecaniza por torneado en forma cilíndrica deseada, 
y por medio de una fresadora, se corta en ella una superficie longitudinal, donde incidirá el láser de bombeo. El resultado final son muestras cilíndricas de $10 \mathrm{~mm}$ de diámetro, con una longitud de $10 \mathrm{~mm}$ y un plano longitudinal de $\sim 4 \mathrm{~mm}$. Las tres caras planas de la muestra que intervienen en el proceso láser, son pulidas manualmente mediante técnicas convencionales, con las que se obtienen superficies con calidades que corresponden a un pulido óptico plano.

Las muestras, una vez mecanizadas y pulidas son bombeadas transversalmente a 534nm con un láser de Nd:KGW (Monocrom STR$2+$ ) doblado en frecuencia que proporciona pulsos 6ns de anchura temporal y 5,5mJ de energía, a una velocidad de repetición de $10 \mathrm{~Hz}$. El sistema de focalización está integrado por tres lentes de cuarzo $\left(\mathrm{L}_{1^{\prime}}\right.$ $\mathrm{L}_{2} \mathrm{y} \mathrm{L}_{3}$ ) que focalizan los pulsos de bombeo formando una línea sobre la cara transversal del cilindro, alcanzándose una frecuencia incidente sobre la muestra de $180 \mathrm{~mJ} / \mathrm{cm}^{2}$. La cavidad resonante está integrada por un espejo plano de aluminio con un $90 \%$ de reflectividad $\left(E_{2}\right)$ y como acoplador de salida se usan las propias caras de la muestra con una longitud de la cavidad de $4 \mathrm{~cm}$ (Figura 1). Se pueden encontrar más detalles sobre el sistema experimental y el sistema de detección en la literatura (25). El error estimado en la medida de la energía es, como máximo, de un $10 \%$.

\section{3.- RESULTADOS Y DISCUSIÓN}

Todas las muestras irradiadas presentaron emisión láser de banda ancha con una duración de pulso de $\sim 5$ ns FWHM. El máximo de emisión láser se encontró comprendido en el intervalo de 560-566nm, dependiendo de la composición del híbrido, con una oscilación del ancho de banda de 7 a $9 \mathrm{~nm}$.

Las eficiencias láser del PM567, definida como la relación entre la energía láser de salida del colorante y la energía incidente de bombeo láser sobre la superficie del cilindro, que se han obtenido para las diferentes formulaciones híbridas sintetizadas aparecen recogidas en la Tabla I. Desde un punto de vista comparativo se han incluido también en la Tabla I los parámetros láser obtenidos para el PM567 cuando éste se encuentra incorporado en copolímeros orgánicos de P(HEMA/ MMA 1/1), sin TEOS, bombeado bajo las mismas condiciones experimentales que las matrices híbridas objeto de este estudio.

TABLA I.- PARÁMETROS LÁSER ${ }^{\wedge}$ OBTENIDOS PARA El PM567 Disuelto EN COPOLÍMEROS DE P(HEMA/MMA 1/1) CON DIFERENTES PROPORCIONES EN PESO DE TEOS. CONCENTRACIÓN DEL COLORANTE: $1,5 \times 10^{-3} \mathrm{M}$. ENERGÍA DE BOMBEO Nd :KGW 5,5 mJ/pulso, VELOCIDAD DE REPETICIÓN: $10 \mathrm{~Hz}$.

\begin{tabular}{|c|c|c|c|c|}
\hline Material & $\lambda_{\max }(\mathbf{n m})$ & $\Delta \lambda(\mathbf{n m})$ & Eficiencia $(\%)$ & ${\text { Vida media: } \mathbf{I}_{\mathbf{n}}(\%)^{\mathbf{b}}}^{\mathbf{b}}$ \\
\hline $0 \%$ TEOS & 561 & 4 & 10 & $25 \%(10000)^{c}$ \\
\hline $5 \%$ TEOS $^{\mathrm{d}}$ & 560 & 8 & 26 & $68 \%(60000)$ \\
\hline $5 \%$ TEOS & 564 & 7 & 22 & $76 \%(60000)$ \\
\hline $10 \%$ TEOS & 566 & 8 & 20 & $11 \%(30000)$ \\
\hline $15 \%$ TEOS & 566 & 9 & 24 & $16 \%(20000)$ \\
\hline
\end{tabular}

$\lambda$ : pico de emisión láser, $\Delta \lambda$ : FWHM de emisión láser.

${ }^{\mathrm{b}}$ Intensidad del láser de salida del colorante después de $n$ pulsos bombeados

(número de pulsos indicado entre paréntesis) referido a la intensidad inicial $\mathrm{I}_{0} ; \mathrm{I}_{\mathrm{n}}(\%)$ $=\left(\mathrm{I}_{\mathrm{n}} / \mathrm{I}_{0}\right) \times 100$

${ }^{\mathrm{c}}$ Muestra sólida bombeada a una velocidad de repetición de $1 \mathrm{~Hz}$ bajo idénticas condiciones experimentales.

${ }^{\mathrm{d}}$ La polimerización se llevó a a cabo en baño termostatizado de agua para ejercer un mayor control sobre la temperatura durante la reacción de síntesis.

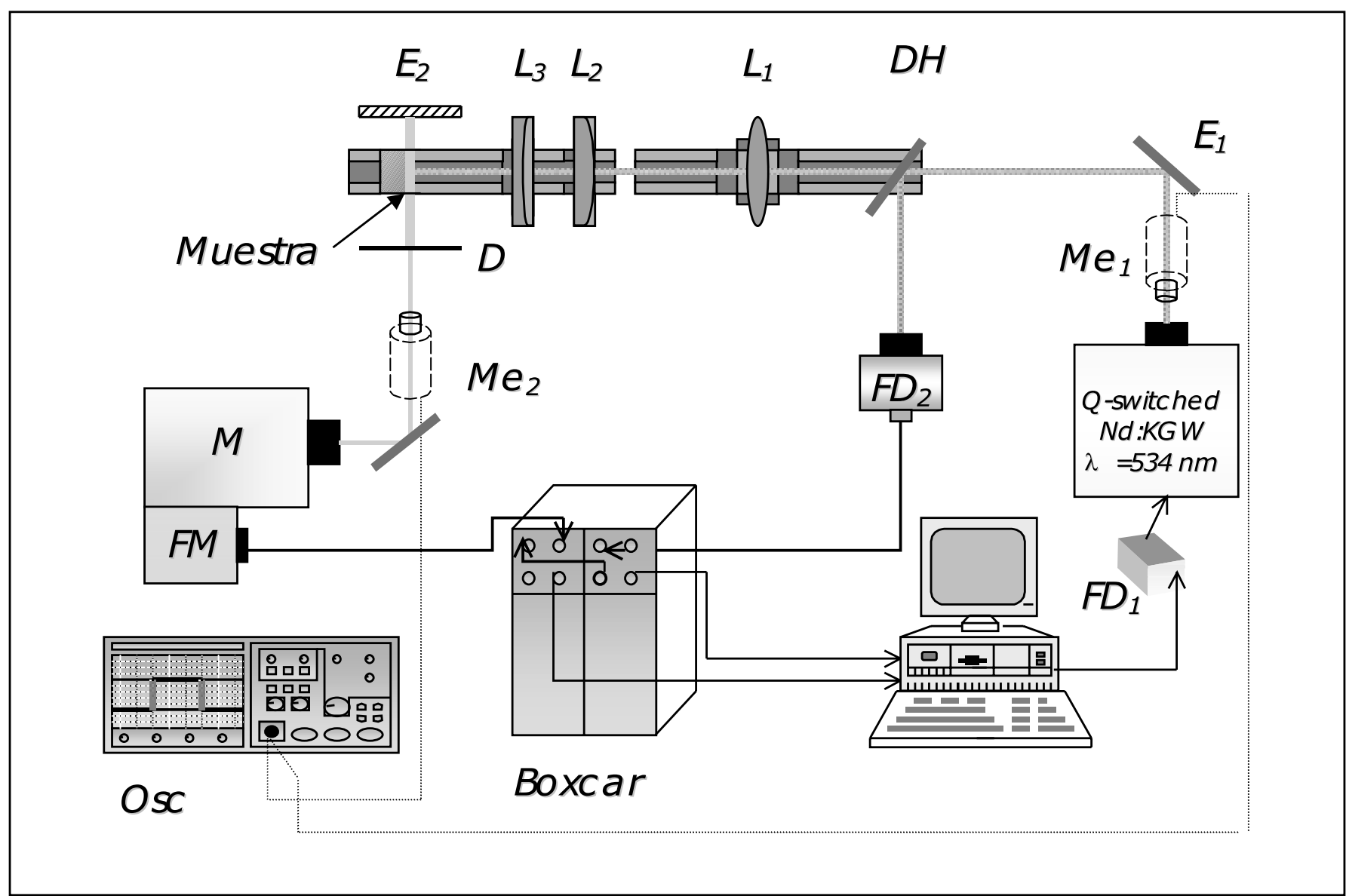

Figura 1.- Montaje experimental del láser, donde $\mathrm{L}_{1}, \mathrm{~L}_{2}$ y $\mathrm{L}_{3}$ son lentes en el sistema de focalización, $\mathrm{Me}_{1}$ y $\mathrm{Me}_{2}$ son medidores conectados al osciloscopio; $\mathrm{D}$ es un diafragma de apertura variable; $\mathrm{E}_{1} \mathrm{y} \mathrm{E}_{2}$ son espejos; $\mathrm{DH}$ es un divisor de haz, $\mathrm{FD}_{1}$ y $\mathrm{FD}_{2}$ son fotodiodos, $\mathrm{M}$ es un monocromador y FM un fotomultiplicador. 
Con el fin de analizar estos resultados desde una perspectiva adecuada se hace necesario comparar las eficiencias láser alcanzadas con las muestras sólidas con aquellas registradas en disoluciones líquidas estáticas de PM567 en acetato de etilo (AcOEt) y etanol (EtOH) (26); disolventes que mimetizan a los monómeros MMA y HEMA, respectivamente; ya que la parte orgánica es el componente mayoritario en estas matrices. Para estas disoluciones, y bajo las mismas condiciones experimentales a que fueron sometidas las muestras sólidas (empleando la misma densidad óptica que en las muestras sólidas y usando $1 \mathrm{~cm}$ de paso óptico en la cara de la celda al acoplador de salida), se registraron eficiencias láser del $33 \%$ y $37 \%$, respectivamente, superiores a las registradas para las muestras híbridas. Es necesario destacar que el acabado final del pulido de las muestras sólidas dista mucho de alcanzar un grado láser, por lo que se podrán alcanzar mejoras significativas en la eficiencia láser desde estas matrices sólidas si se consigue mejorar la calidad óptica de sus superficies. La eficiencia láser de las muestras, dentro del error experimental, y de acuerdo con los datos de la Tabla I, no depende significativamente de la proporción de TEOS, aunque parece claro que la presencia de TEOS, aún en bajas proporciones molares, mejora la eficiencia láser de las muestras respecto a la que se obtiene para el PM567 incorporado en matrices copoliméricas de P(HEMA/MMA 1/1) que no incluyen TEOS en su composición.

Las eficiencias láser alcanzadas en este trabajo mejoran algunos de los resultados obtenidos anteriormente por otros autores cuando el PM567 es incorporado en matrices inorgánicas e híbridas, por ejemplo, cuando se dopan vidrios de Polycom con PM567, las eficiencias láser recogidas son del 5\% y 16\% bajo condiciones de desoxigenación y de saturación de aire, respectivamente (23). Sin embargo, también han sido descritas mayores eficiencias para el PM567 en xerogeles, en cuyo caso la eficiencia láser obtenida variaba desde el $29 \%$ hasta alcanzar valores del $66 \%$, dependiendo del tipo de cavidad óptica de colorante empleada y de los parámetros de bombeo láser $(16,17)$. Es importante resaltar el hecho de que en todos los casos referenciados el bombeo de láser era longitudinal y la reflectividad del acoplador de salida estaba optimizada, mientras que los resultados obtenidos en el presente trabajo resultan de bombeo láser transversal, sin optimizar la cavidad resonante.

Para todas las matrices híbridas descritas se ha estudiado la evolución de la eficiencia láser en función del número de pulsos bombeados sobre una misma muestra en posición fija a una velocidad de repetición de $10 \mathrm{~Hz}$. Los resultados aparecen reflejados en la Tabla I, mientras en la Figura 2 se muestra gráficamente la fotoestabilidad del PM567 incorporado a matrices basadas en P(HEMA/MMA 1/1) incluyendo un $5 \%$ p y $10 \%$ p de TEOS. Inicialmente, la fotoestabilidad se incrementa significativamente al aumentar la proporción de TEOS en la matriz. Este efecto puede ser atribuido a un aumento en la disipación térmica que ofrece el material híbrido, que hace que se vea sensiblemente reducida la degradación térmica del colorante bajo irradiación láser sin que a su vez exista pérdida de los beneficios que supone una matriz polimérica para el mismo. Sin embargo, cuando la proporción de TEOS aumenta por encima del $5 \%$ en peso, el resultado es un drástico descenso de la vida útil del material, si bien su vida media sigue siendo suficientemente alta y comparable a la que se obtiene desde una matriz puramente orgánica de $\mathrm{P}$ (HEMA/MMA 1/1).

Si bien es muy probable que no exista una única causa capaz de explicar en su totalidad el comportamiento de estas matrices, parece ser que, en una primera aproximación, la proporción del componente inorgánico presente en la matriz juega un papel decisivo en la fotodegradación del colorante, cuyo efecto inmediato es que una fracción de moléculas del colorante se están transformando en especies activas (tripletes, radicales) capaces de reaccionar con otras moléculas cercanas de colorante, impurezas, radicales y grupos presentes en las cade-

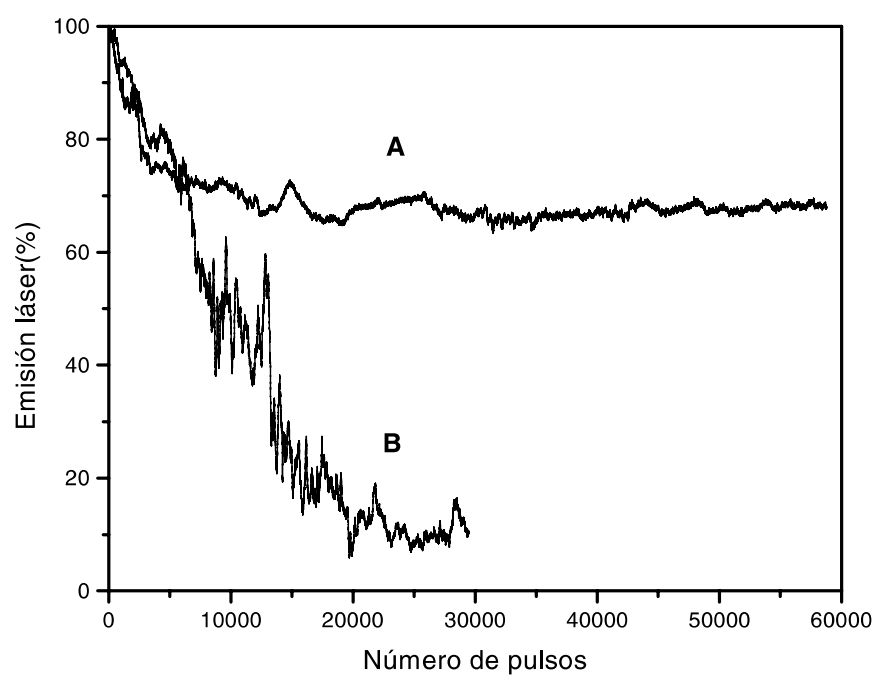

Figura 2.- Emisión láser normalizada en función del número de pulsos para PM567 incorporado en A) P(HEMA/MMA 1/ 1+5\% p TEOS) y B) P(HEMA / MMA 1/1+10\% p TEOS). Concentración de colorante: $1,5 \times 10^{-3} \mathrm{M}$. Energía de bombeo y velocidad de repetición: 5,5 mJ/ pulso y $10 \mathrm{~Hz}$, respectivamente.

nas poliméricas o cualquier otra especie activa presente en el medio. De hecho, un incremento de la proporción inorgánica en el material híbrido hace que aumente considerablemente la acidez remanente del medio, lo cual puede favorecer la efectividad y probabilidad de estos procesos reactivos. Además, debido a que el mecanismo fotoquímico debe ser, al menos, bimolecular, el proceso debe ser altamente dependiente de la microestructura y flexibilidad del material.

Los resultados anteriormente expuestos indican que, con objeto de aumentar la fotoestabilidad del colorante/sistema híbrido, tendremos que llegar a una situación de compromiso entre la mejora de la disipación térmica del material y el incremento de la degradación fotoquímica del colorante, simplemente variando la composición de los componentes orgánico-inorgánico del material. En el caso que nos ocupa, el resultado mas satisfactorio lo hemos alcanzado con la muestra híbrida P(HEMA/MMA 1/1+ 5\% p TEOS), en cuyo caso la emisión láser se mantiene en el $76 \%$ de su valor inicial aún después de 60000 pulsos a una velocidad de repetición de $10 \mathrm{~Hz}$. Este resultado representa, según nuestro actual conocimiento del tema, la mejor fotoestabilidad láser alcanzada hasta la fecha para el colorante PM567 incorporado en matrices inorgánicas e híbridas, aún cuando éstas últimas hayan sido bombeadas en condiciones más favorables que en nuestro caso, es decir, por bombeo longitudinal y a más bajas velocidades de repetición $(1 \mathrm{~Hz})(15,21,23)$. Además, estos resultados suponen una mejora de los tiempos de vida láser del PM567 incorporado a matrices orgánicas, donde la emisión láser de este colorante disuelto en matrices entrecruzadas y lineales de MMA con diferentes co-monómeros y bombeadas bajo las mismas condiciones experimentales mantienen un $80 \%$ de emisión láser después de 45000 pulsos a una velocidad de repetición de $10 \mathrm{~Hz}$ (27).

Recientemente, Yang y colaboradores han publicado la fotoestabilidad del PM567 dopado en monolitos de ORMOSIL con pérdidas de la emisión láser por debajo del 15\% después de 106000 pulsos (28). Comparando con los resultados que mostramos, hay que tener en cuenta que esta vida media láser se consiguió prolongar incrementando el espesor de la muestra sólida al bombearla longitudinalmente con una fluencia de $0.1 \mathrm{MJ} / \mathrm{cm}^{2}$ y a una velocidad de repetición de $2 \mathrm{~Hz}$.

Teniendo en cuenta que la reacción de síntesis de estos materiales híbridos es un proceso altamente exotérmico, consideramos de interés el analizar la influencia que el incremento inherente de temperatura, 
que puede autoacelerar la velocidad del proceso, tiene en la emisión láser del material sólido final. Por este motivo, llevamos a cabo la síntesis de PM567 incorporado a matrices de P(HEMA/MMA 1/1+5\%p TEOS) de forma que pudiéramos ejercer un estricto control de la temperatura de polimerización mediante el enfriamiento continuo por agitación del medio circundante a la muestra. Según los datos recogidos en la Tabla I, podemos concluir que la exotermicidad de la reacción no ejerce ninguna influencia significativa ni sobre la eficiencia ni sobre la fotoestabilidad láser del material. En consecuencia, el proceso de polimerización y el tratamiento térmico correcto de la muestra podrá llevarse a cabo empleando una metodología que permite un procesado de un mayor número de muestras de forma simultanea.

Con objeto de alcanzar un mayor conocimiento acerca de la fotoestabilidad de estos nuevos materiales, llevamos a cabo un estudio de la dependencia del tiempo de vida media láser en función de la velocidad de repetición de bombeo sobre las mismas. En la Figura 3 podemos observar el efecto que la velocidad de repetición de bombeo (a 5 y $10 \mathrm{~Hz}$ ) produce en la fotoestabilidad del PM567 incorporado a matrices de P(HEMA/MMA 1/1+15\% p TEOS). Observamos como al aumentar la velocidad de bombeo, la velocidad de degradación del material en estudio se incrementaba significativamente. Parece pues, que a mayores velocidades de repetición, los canales de disipación de la energía liberada al medio en forma de calor no son suficientemente efectivos y, en consecuencia, se incrementa la degradación térmica del colorante. Estos resultados están en concordancia con los estudios previos llevados a cabo sobre el efecto que el calentamiento del medio tiene en la estabilidad de láseres de colorante poliméricos, que evidenciaban que la acumulación de calor local dentro del material se incrementa significativamente a velocidades de bombeo superiores a $1 \mathrm{~Hz}(29)$.

En resumen, se ha conseguido incorporar PM567 a materiales híbridos obtenidos a partir de alcóxidos de silicio modificados orgánicamente. Los primeros resultados que hemos obtenido con estos nuevos materiales resultan muy prometedores, y demuestran que la adición de pequeñas proporciones de TEOS en copolímeros de P(HEMA/ MMA 1/1) favorecen la disipación de calor reduciendo la degradación térmica del colorante $\mathrm{y}$, consecuentemente, mejorando significativamente su acción láser. De hecho, algunos de los resultados alcanzados con estos nuevos materiales mejoran, en mucho, las fotoestabilidades alcanzadas para el PM567 incorporado en otras matrices inorgánicas e

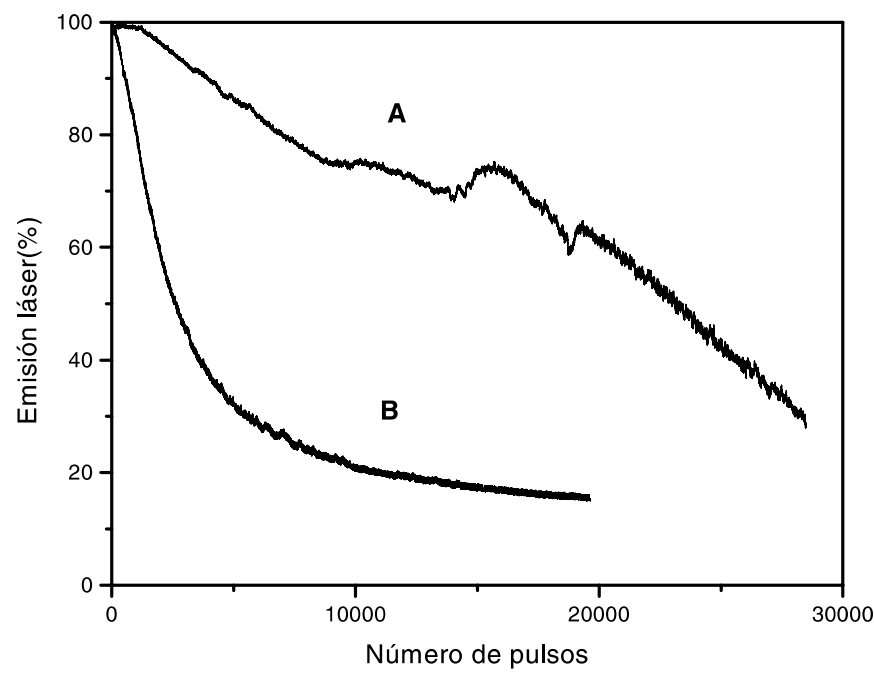

Figura 3.- Influencia de la velocidad de repetición de bombeo láser $(\mathrm{A}=5 \mathrm{~Hz}$ y $\mathrm{B}=10 \mathrm{~Hz})$ en la fotoestabilidad láser del colorante PM567 incorporado en P(HEMA/MMA 1/1 + 15\% p TEOS). Concentración del colorante: $1,5 \times 10^{-3} \mathrm{M}$. Energía de bombeo: $5,5 \mathrm{~mJ} /$ pulso híbridas, así como, y siempre teniendo en cuenta las condiciones experimentales de bombeo láser, mejoran o al menos igualan a muchas de las matrices orgánicas que ya han sido utilizadas como hospedadores para este colorante.

Estos resultados indican que un láser de colorante en estado sólido basado en estas nuevas matrices híbridas orgánico-inorgánicas podrían llegar a reemplazar a algunos sistemas láser líquidos en algunas aplicaciones concretas.

\section{4.- AGRADECIMIENTOS}

Este trabajo está financiado por un proyecto MAT2000-1361-C0401 del CICYT.

O. García y C. Gómez desean agradecer a la Comunidad Autónoma de Madrid (CAM) la financiación de su beca postdoctoral.

\section{5.- BIBLIOGRAFÍA}

1. W. Thomas (ed.): "Handbook of photographic science and engineering", J. Wiley, New York (1973).

2. M.J. Bowden, S.R. Turner: "Polymers for electronic and photonics", ACS, (1987)

3. C.G. Roffrey: "Photopolymerization of surface coatings", Wiley, New York, (1982).

4. H.I. Bjelkhagen (ed.): "Selected papers on holographic recording materials", SPIE Milestone Series, SPIE, Vol. MS 130, Bellingham, Washington, (1996).

5. P.K.T. Oldring (ed.): "Chemistry and technology of uv and eb formulations for coatings inks and paints", (Sita Technol., Vol. 1-5, Londres, UK, (19911994).

6. L.A. Linden, "Applied photochemistry in dental science", Proc. Indian. Acad. Sci. (Chem. Sci.), 105(6), 405-419 (1993).

7. P. Suppan: "Chemistry and light", Royal Soc. of Chemistry, Cambridge, (1994).

8. J.M. Kelly, C.B. McArdle, M.J.F. Maunder: "Photochemistry and polymer systems", Royal Soc. of Chemistry, Cambridge, (1995).

9. V.V. Krongauz, A.D. Trifunac: "Processes in photoreactive polymers", Chapman and Hall, London, (1995).

10. P. Judeinstein y C. Sánchez, “Hybrid organic-inorganic materials: a land of multidisciplinarity", J. Mater. Chem., 6(4), 511-525 (1996)

11. L.L. Hench y J.K. West, "The sol-gel process", Chem. Rev., 90(1), 33-72 (1990).

12. D.L. Wise, G.E. Wnek, D.J. Trantolo, T.M. Cooper y J.D. Gresser Eds., en "Photonic polymer systems: fundamentals, methods and applications," Marcel Decker, Inc., New York, capítulo 12, pp. 437-480 (1998).

13. C. Sánchez y F. Ribot, “Design of hybrid organic-inorganic materials synthesized via sol-gel chemistry", New J. Chem., 18(10), 1007-1047 (1994).

14. J.C. Altman, R.E. Stone, B. Dunn, F. Nishida, "Solid-state laser using a rhodamine-doped silica gel compound", IEEE Photon. Tech. Lett. 3(3), 189-190 (1991).

15. M. Canva, P. Georges, J.F. Perelgritz, A. Brum, F. Chaput, J.P. Boilot; “Perylene-and pyrromethene-doped xerogel for a pulsed laser", Appl. Opt. 34(3), 428-431 (1995).

16. R.E. Hermes, T.H. Allik, S. Chandra, J.A. Hutchinson; “High-efficiency pyrromethene doped solid-state dye lasers", Appl. Phys. Lett. 63(7), 877-879 (1993).

17. A. Costela, I. García-Moreno, J.M. Figuera, F. Amat-Guerri, R. Mallavía, M.D. Santa-María, R. Sastre; “Solid-state dye lasers based on modified rhodamine 6G dyes copolymerized with methacrylic monomers", J. Appl. Phys. 80, 31673173 (1996)

18. K.M. Dyumaev, A.A. Manenkov, A.P. Maslyukov, G.A. Matyushin, V.S. Nechitailo, A.M. Prokhorov, "Dyes in modified polymers: problems of photostability and conversion efficiency at high intensities", J. Opt. Soc. Am. B , 9, 143-151 (1992).

19. F.J. Duarte, "Solid-state multiple-prism grating dye laser oscillators", Appl. Opt. 33, 3857-3860 (1994).

20. R. Sastre, A. Costela; "Polymeric solid-state dye lasers", Adv. Mater. 7, 198204 (1995).

21. M. Faloss, M. Canva, P. Georges, A. Brun, F. Chaput, J.P. Boilot, “Toward millions of laser pulses with pyrromethene- and perylene-doped xerogels", Appl. Opt., 36, 6760-6763 (1997). 
22. T.H. Allik, S. Chandra, T.R. Robinson, J.A. Hutchinson, G. Sathyamoorthi, J.H. Boyer, "Laser performance and material properties of a high temperature plastic doped with pyrromethene-BF2 dyes", Mater. Res. Soc. Symp. Proc., 329, 291-296 (1994)

23. M.D. Rahn, T.A. King, A.A. Gorman, I. Hamblett, "Photostability enhancement of Pyrromethene 567 and Perylene Orange in oxygen-free liquid and solid dye lasers", Appl. Opt., 36(24), 5862-5871 (1997).

24. P. Hajjí, L. David, J.F. Gerard, J.P. Pascault y G. Vigier, "Synthesis, structure, and morphology of polymer-silica hybrid nanocomposites based on hydroxyethyl methacrylate", J. Polym. Sci., Part B: Polym. Phys., 37(22), 3172-3187 (1999).

25. M. Rodríguez, A. Costela, I. García-Moreno, F. Florido, J.M. Figuera, R. Sastre, "Automatized control system for solid-state dye-lasers", Meas. Sci. Technol., 6, 971-978 (1995).
26. A. Costela, I. García-Moreno, J. Barroso, R. Sastre, "Laser performance of pyrromethene 567 dye in solid matrices of methyl methacrylate with different comonomers", Appl. Phys. B, 70, 367-373 (2000).

27. A. Costela, I. García-Moreno, C. Gómez, O. García , R. Sastre, “Laser performance of pyrromethene 567 dye in solid polymeric matrices with different cross-linking degrees", J. Appl. Phys., 90, 3159-3166 (2001).

28. Y. Yang, G. Qian, Z. Wang, M. Wang, "Influence of the thickness and composition of the solid-state dye laser media on the laser properties", Opt. Commun., 204, 277-282 (2002).

29. R. Duchowicz, L.B. Scaffardi, A. Costela, I. García-Moreno, R. Sastre, A.U. Acuña, "Photothermal characterization and stability analysis of polymeric dye lasers", Appl. Opt., 39, 4959-4963 (2000)

Recibido: 1.2.03

Aceptado: 30.11 .03 